For Physical Review B, Rapid Comunications

\title{
Ordering of self-diffusion barrier energies on $\mathrm{Pt}(110)-1 \times 2$
}

\author{
Peter J. Feibelman \\ Sandia National Laboratories \\ Albuquerque, NM 87185-1413
}

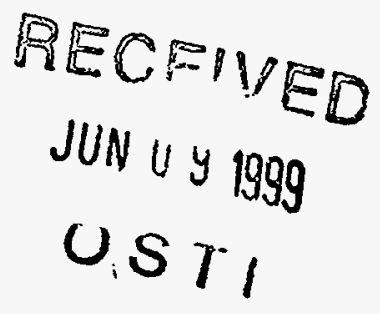

Bond-counting arguments, supported by ab-initio calculations, predict a lower barrier for "leapfrog" diffusion of Pt addimers on Pt(110)-1x2 than for adatom diffusion or addimer dissociation. This conflicts with experiment, possibly signalling contaminant influence.

Controlling the time-evolution of surface morphology requires understanding how surface species migrate. High-resolution microscopy provides snapshots of diffusion paths, but hard-to-capture details can make their interpretation a challenge. This is important because the mechanisms that dominate matter-transport on a surface need not be "obvious" ones. One might guess, e.g., that self-diffusion on "missing row" $\mathrm{Pt}(110)-1 \times 2$ would be one-dimensional, but the minimum-energy path (MEP) for a diffusing Pt adatom need not be parallel to a channel. It might instead lead to the top of a wall and along it before returning to the channel bottom, as in Fig. $1 .^{1}$

Adatom displacement from channel bottom to top is known to permit facile migration of Pt-adclusters on Pt(110)-1x2. Notwithstanding the frequent assumption that only isolated adatoms displace at low $\mathrm{T}$, Linderoth, et al. find that even trimers and tetramers migrate on this surface at $300-350 \mathrm{~K} .^{2}$ They show that the Pt at the end of a cluster moves up to a site between the rest of the cluster and the channel 


\section{DISCLAIMER}

Portions of this document may be illegible in electronic image products. Images are produced from the best available original document. 
For Physical Review B, Rapid Comunications

wall. It hops along the cluster, then returns to the channel at its other end. Called "leapfrog diffusion," the same process occurs in molecular-dynamics simulations by Montalenti and Ferrando. ${ }^{3}$

The energetic advantage of leapfrogging is that on the MEP all adatoms but one occupy high-coordination channel sites. This advantage, however, is shared by a dissociation-recombination mechanism in which atom 1 at one end of a cluster separates from the remainder, then, successively, atom 2 catches up to 1 , atom 3 catches up to the 1-2 dimer, etc., and eventually the reconstituted cluster is displaced.

The focus of this note is the recent Scanning Tunneling Microscopy (STM) observation ${ }^{4}$ that dissociation-recombination is not just facile, but the most facile dimer-diffusion mode at 300-350K. I show that this result conflicts with bondcounting arguments and ab-initio calculations; both favor leapfrogging over adatom diffusion and addimer dissociation. The barriers I compute for dimer dissociation $(1.13 \mathrm{eV})$ and leapfrogging $(0.76 \mathrm{eV})$ imply, e.g., that the former is $10^{6}$ times less probable at $300 \mathrm{~K}$ than dimer leapfrogging, assuming equal prefactors. ${ }^{5}$ Thus $a b$ initio theory suggests revisiting the experimental analysis.

Physical arguments - Leapfrog diffusion of dimers should be more facile than monomer diffusion because the MEP's for both processes involve moving an adatom from its initial channel-bottom site up a channel wall, along it, then down again (cf. Figs. 1 and 2). The key distinction is that in the leapfrog case the moving adatom always has one additional neighbor, its partner adatom. Since binding-energy loss per broken bond varies inversely with the initial number of neighbors, ${ }^{6}$ barriers along the leapfrog.path must therefore be lower. 
For Physical Review B, Rapid Comunications

The argument comparing dimer leapfrog diffusion with dissociation begins from an estimate of the dissociation barrier. At such a barrier, one adatom remains in its initial high-coordination hollow while the other is at a bridge adjacent to its initial site. On $\mathrm{Pt}(110)-1 \times 2$, this bridge may lie directly down the missing row or on one of the (111)-facets that form the missing-row walls. Either way, the adatom at the bridge is far enough from the one that stays behind that their interaction is small. Thus, the dimer-dissociation barrier energy $\approx$ the adatom-adatom binding energy plus the monomer diffusion barrier. Since the inter-adatom binding energy is independent of dissociation path, the bridge-site energy determines the most facile dissociation path. Displacement of an adatom up a channel wall is therefore the most facile monomer-diffusion and dimer-dissociation mode.

This implies that the addimer configuration illustrated in Fig. 2 is a "doorway state" through which both dissociation and leapfrog-diffusion MEP's pass. That the barrier to dissociation is higher than to leapfrogging is a corollary. To see this, imagine removing atom 2 from the picture. By symmetry, there is then no energetic difference between displacing atom $\mathbf{1}$ to either $f c c$ site $\mathbf{f}$ or $\mathbf{f}^{\prime}$, as there is if atom $\mathbf{2}$ is present. In the latter case, atom $\mathbf{1}$ breaks its bond to atom $\mathbf{2}$ in displacing to site $\mathbf{f}$, while it does not in moving to site f'. Moving to $f^{\prime}$ is therefore less costly.

Numerics - To flesh out these arguments, I have computed ab-initio activation barriers using VASP (Vienna ab-initio simulation package), ${ }^{7-9}$ its corresponding ultrasoft-pseudopotential data-base, ${ }^{10}$ and the Perdew-Wang '91 Generalized Gradient Approximation (GGA). ${ }^{11}$ Plane-wave calculations for first-row atoms and transition metals typically require unwieldy basis sets, but the ultrasoft pseudopotential assures convergence for Pt with the modest basis-cutoff of $14.7 \mathrm{Ry}$, making the present work tractable despite involving large unit cells. 
For Physical Review B, Rapid Comunications

I represent the missing-row surface of $\operatorname{Pt}(110)$ via an 8-layer slab, thin enough to moderate computational cost, but not so thin that quantum-size artifacts should be important. [Note: an 8-layer (110) slab is only as thick as a 5-layer (111) slab.] I constrain the atoms of the lower three layers to bulk GGA crystal locations, remove every second [1]0] row of $\mathrm{Pt}$ atoms on the upper surface to create the $1 \times 2$ missingrow reconstruction, and relax the remaining atoms to optimize the GGA functional. To speed electronic relaxation, I use Methfessel and Paxton's Fermi-level smearing (width $=0.3 \mathrm{eV}$ )..$^{12}$ I set the lattice parameter of the model slab to the optimal bulk GGA value, $3.99 \AA$ (experiment $=3.92 \AA$ ).

Computing barriers begins with a determination of the adspecies' optimal binding geometries. Incidentally, the results allow comparison to Linderoth, et al.'s estimate of the dimer dissociation energy, $70 \mathrm{meV}$, based on statistical analysis of STM micrographs. ${ }^{4}$

I perform the geometry optimizations in $\mathrm{Pt}(110)-4 \times 4$ unit cells (cf. Fig. 2). In each cell there are two missing rows, one empty and one occupied by adatoms. Thus, adatoms in different missing rows interact weakly. Each cell extends four PtPt spacings along the $[1 \overline{1} 0]$ rows. This reduces interaction in the same row. I sample the Surface Brillouin Zone with a rectangular array of eight k's, two in the [001] direction by four in the [1ํㅣㅇ.

Adatoms optimally occupy channel hollows coordinated to five slab atoms, and to each other in the case of an addimer. Computed interaction energies, summarized in Table 1, correspond to the formula, $E_{\text {diss }}=2 E_{\text {monomer }}-E_{\text {clean }}-E_{\text {dimer }}$ where $E_{\text {dimer }}$ and $E_{\text {monomer }}$ are the total energies corresponding to adspecies in the $4 \times 4$ cell and $E_{\text {clean }}$ is the energy per $4 \times 4$ cell of the clean, missing-row surface. 
For Physical Review B, Rapid Comunications

Table 1 reveals two important results: 1) to separate a nearest-neighbor dimer costs $0.21 \mathrm{eV}$, about 3 times more than is derived from STM data in Ref. 4, and 2) this cost is all expended in separating a pair of adatoms to next-nearest-neighbor hollows, i.e., the interaction between adatoms dies off very rapidly (as assumed in Ref. 4). The disagreement between the measured and computed dimer dissociation energies is "only" $0.14 \mathrm{eV}$. It is, however, the first of several disagreements with measurement, which together point to a systematic problem of some kind.

To compute transition-geometries and barrier energies, I use Jónsson's Nudged Elastic Band (NEB) method, ${ }^{13}$ which, starting from replicas that interpolate between initial and final atomic configurations, relaxes them to an approximate MEP. The method requires using enough replicas to allow a good approximation to the tangent to the MEP. Thus one can nullify the effect on each replica of the force along the local tangent and prevent them from falling away from the saddle point of interest.

Here, when four replicas do not produce a smooth MEP upon relaxation, it is because the path passes through an intermediate local minimum. I then either add replicas or break the MEP determination into two parts. Barrier energies quoted are numerically accurate to $\sim 20 \mathrm{meV}$.

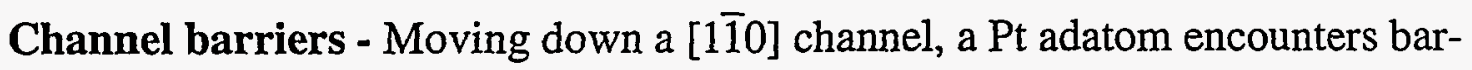
riers at 2-fold "long-bridges." The high computed barrier, $1.03 \mathrm{eV}$ (cf. Table 2), reflects the substantial loss of coordination suffered when the Pt leaves its 5-fold channel hollow. At the barrier, the Pt's second neighbors are $2.91 \AA$ away compared to nearest neighbors at $2.53 \AA$. For reference, the GGA Pt-Pt nearest-neighbor separation is $2.82 \AA$. So describing the transition state as two-fold coordinated is accurate. 
For Physical Review B, Rapid Comunications

Recall that the estimated energy needed to displace one adatom of a dissociating dimer to the in-channel barrier is the sum of the energy to displace a monomer from 5-fold hollow to 2-fold bridge, $1.03 \mathrm{eV}$, plus the energy to break the dimer bond, $0.21 \mathrm{eV}$. This estimate, $1.24 \mathrm{eV}$, is precisely what a direct NEB dissociation barrier calculation yields (cf. Table 1).

Wall barriers - The MEP for a monomer moving from a channel hollow to one of the four nearest $f c c$ channel-wall sites (labelled $\mathbf{f}$ in Fig. 1) passes through a wall $h c p$ hollow (labelled $\mathbf{h}$ in Fig. 1). There are thus two barriers along the MEP. The first, between the channel hollow and the $h c p$ site equals $0.88 \mathrm{eV}$, the second, between the $h c p$ and $f c c$ sites, amounts to $0.93 \mathrm{eV}$. The minimum barrier for monomer diffusion along the $\mathrm{Pt}(110)-1 \times 2$ missing row surface is thus $0.93 \mathrm{eV}$, in agreement with previous calculations by Lorensen, et al., ${ }^{1}$ but $0.12 \mathrm{eV}$ higher than Linderoth, et al.'s experimental value. ${ }^{4,14}$

As discussed above, Fig. 2 illustrates a "doorway" through which both dimer dissociation and leapfrog diffusion MEP's pass. Its energy is $0.67 \mathrm{eV}$ relative to the dimer ground state. According to NEB path relaxation, the barrier separating these two configurations is $0.76 \mathrm{eV}$, which, as predicted on physical grounds, is lower than the $0.88 \mathrm{eV}$ barrier for a monomer to move from a channel hollow to a wall $h c p$ site.

From the doorway, dimer dissociation occurs if the adatom in the $h c p$ wall site moves through the bridge between atoms $t_{1}$ and $w$ of Fig. 2 , to the fcc hollow labelled $\mathbf{f}$. The computed barrier, $1.13 \mathrm{eV}$, is again $\approx$ the dimer dissociation energy, $0.21 \mathrm{eV}$ plus the energy of a monomer at the 2 -fold bridge, $0.93 \mathrm{eV}$. Thus, as expected, dissociation on the wall is preferred to dissociation in the channel. 
For Physical Review B, Rapid Comunications

A less costly outcome is a leapfrog diffusion event. Now adatom 1 proceeds over a low ( $20 \mathrm{meV})$ barrier, from the $h c p$ site where it resides in Fig. 2, to $f c c$ wall site f' where the system energy is only $0.31 \mathrm{eV}$ above that of the dimer ground state. The low energy of this configuration can be attributed to passivation of two undercoordinated $\mathrm{Pt}$ atoms $\left(\mathrm{t}_{1}\right.$ and $\left.\mathbf{t}_{2}\right)$ at the top of the channel wall.

Adatom 1 then squeezes between adatom 2, and $t_{2}$, to $f c c$ site $\mathbf{f}$ ', before returning to the channel bottom. The barrier along the path between sites $f$ and $f$ ' is only $0.72 \mathrm{eV}$, reflecting not only the energetic gain associated with passivating undercoordinated neighbors, but also the ability of such neighbors to relax elastically.

Theory vs. Experiment - The barriers computed here are in qualitative conflict with what Linderoth, et al. derive from statistical analysis of their STM movies. ${ }^{4}$ Theory implies that leapfrog dimer-diffusion, whose barrier is only $0.76 \mathrm{eV}$, should proceed much more rapidly, at low $\mathrm{T}$, than either monomer diffusion or dissociation-recombination-mode dimer diffusion, whose minimum barriers are 0.93 and $1.13 \mathrm{eV}$. In contrast, assuming normal prefactors for all processes, Ref. 4 says that dissociation-recombination is the most facile dimer diffusion process, with a slightly higher barrier, $0.84 \mathrm{eV}$, than that for monomer diffusion, $0.81 \mathrm{eV}$.

How these differences can be reconciled? With the computed barrier ordering and physical arguments in accord, the focus turns to experimental concerns. An obvious one to consider is contamination.

CO? - The Aarhus group has not neglected the possible influence of surface contaminants in their diffusion studies. Recently, they reported direct STM observation of adsorbed $\mathrm{H}$ jumping onto Pt adatoms on Pt(110)-1x2, causing the Pt's to displace unusually quickly. ${ }^{15}$ 
For Physical Review B, Rapid Comunications

Given that $\mathrm{H}$ can be excluded by observation, the obvious candidate contaminant is $\mathrm{CO}$, a common gas in UHV systems whose STM signature is unknown. Unfortunately, calculations are more time-consuming with $\mathrm{CO}$ in the unit cell. $\mathrm{C}$ and $\mathrm{O}$ have harder pseudopotentials than $\mathrm{Pt}$, requiring use of a bigger plane-wave basis. Also, each $\mathrm{CO}$ molecule adds six degrees of freedom to the configuration space to be searched for minima and saddles.

I have begun by asking by how much an ad-CO reduces the energy needed to dissociate a Pt ad-dimer on Pt(110)-1x2? The results (see Table 4) suggest that pursuing the question of CO-effects would be worthwhile.

To estimate how a single $\mathrm{CO}$ affects the dissociation energy of a $\mathrm{Pt}$ ad-dimer, $E_{\text {diss }}$ I use the formula, $E_{\text {diss }}=E_{\text {monomer }+C O}+E_{\text {monomer }}-E_{\text {clean }}-E_{\text {dimer }+C O} \cdot$ On the right-hand-side of this formula, the first two terms are the energies of two $\operatorname{Pt}(110)$ slabs, one with a Pt adatom and one with a CO-covered Pt adatom in each supercell. In last two, the Pt adatom from the first of the initial slabs has been moved to the second, forming a $\mathrm{Pt}$ ad-dimer with a single $\mathrm{CO}$ attached to it and leaving a clean $\operatorname{Pt}(110)-1 \times 2$ slab behind. $E_{\text {diss }}$ is then the energy gained in forming the dimer. The numerical results (see Table 4) are for calculations in the same $4 \times 4$ supercells as the clean $\mathrm{Pt}(110)$ results of Tables 1-3. But I now use a 29.1 Ry plane-wave cutoff, to allow for the rapid spatial variation of the $\mathrm{O}(2 \mathrm{p})$ orbital.

The results are as follows: A single $\mathrm{CO}$ prefers to bind to a Pt ad-dimer atop one of the dimer adatoms; binding at the dimer bridge is less favorable by $0.11 \mathrm{eV}$. In what may just be a suggestive coincidence, the addimer dissociation energy, starting with the $\mathrm{CO}$ in the atop geometry, is $73 \mathrm{meV}$. This value, about $1 / 3 E_{\text {diss }}$ for a clean ad-dimer, is rather close to the $70 \mathrm{meV}$ derived in Ref. 4 from statistical analysis of STM movies of dissociation and re-association. 
For Physical Review B, Rapid Comunications

In deciding if this agreement is more than a coincidence, one must consider that a dimer with a single $\mathrm{CO}$ firmly bound to one of its $\mathrm{Pt}$ atoms should not look symmetric, while Ref. 4's dimer images do. The reason might be that only a small barrier, $\sim 0.16 \mathrm{eV}$ according to NEB calculations, impedes displacement of a $\mathrm{CO}$ from the favored atop binding site to the dimer bridge. Thus, at $300 \mathrm{~K}$ a $\mathrm{CO}$ will frequently hop from one end of a dimer to the other, or be dragged by a scanning probe tip. An alternate explanation of the observed symmetry is that dimers always bind two CO's. Calculations indicate, however, that two CO's on an addimer repel strongly enough to destabilize it.

To recapitulate, the ordering of dissociation and diffusion barriers for Pt adatoms on clean $\mathrm{Pt}(110)-1 \times 2$ is susceptible to simple bond-counting arguments. Systematic disagreement of the predicted ordering with experiment suggests revisiting the experimental analysis. Numerical results hint, though far from prove, that $\mathrm{CO}$ contamination effects may be significant. Needless to say, if $\mathrm{CO}$ is not only omnipresent, but has important effects on the time-evolution of Pt surfaces even at minute coverages, we must understand and document them.

Acknowledgments - I thank F. Besenbacher and S. Horch for fruitful discussions. VASP and its pseudopotentials were developed at the Institut für Theoretische Physik of the Technische Universität Wien. Sandia is a multi-program laboratory operated by Sandia Corporation, a Lockheed-Martin Company, for the U. S. Department of Energy under Contract No. DE-AC04-94AL85000. 
For Physical Review B, Rapid Comunications

\section{References -}

1. H. T. Lorensen, J. K. Nørskov and K. W. Jacobsen, to be published.

2. T.R. Linderoth, et al., Phys. Rev. Lett. 82, 1494(1999).

3. F. Montalenti and R. Ferrando, Phys. Rev. Lett. 82, 1498(1999).

4. T.R. Linderoth, et al., Surf. Sci. 402-404, 308(1998), and unpublished.

5. L. Petersen, private communication.

6. See, e.g., P. Stoltze, J. Phys. Cond. Mat. 6, 9495(1994), Fig. 1.

7. G. Kresse and J. Hafner, Phys. Rev. B47, 558 (1993); ibid. B49, 14251 (1994).

8. G. Kresse and J. Furthmüller, Comput. Mat. Sci. 6, 15 (1996).

9. G. Kresse and J. Furthmüller, Phys. Rev. B54, 11169 (1996).

10. D. Vanderbilt, Phys. Rev. B41, 7892(1990); A. Pasquarello, et al., Phys. Rev. Lett. 69, 1982(1992); K. Laasonen, et al., Phys. Rev. B47, 10142(1993); G. Kresse and J. Hafner, J. Phys.: Cond. Mat. 6, 8245(1994).

11. J. P. Perdew, in Electronic Structure of Solids '91, edited by P. Ziesche and H. Eschrig (Akademie Verlag, Berlin, 1991); J. P. Perdew and Y. Wang, unpublished. 12. M. Methfessel and A. T. Paxton, Phys. Rev. B40, 3616(1989).

13. H. Jónsson, G. Mills, K. W. Jacobsen, in Classical and Quantum Dynamics in Condensed Phase Simulations, ed. B. J. Berne, G. Ciccotti and D. F. Coker (World Scientific, 1998).

14. Using the new GGA of B. Hammer, Phys. Rev. B59, 7413(1999), Lorensen, et al. (Ref. 1) obtain better agreement with Ref. 4's barrier, but whether this GGA generally improves structural energetics is not yet known.

15. S. Horch, et al., NATURE 398, 134(1999). 
For Physical Review B, Rapid Comunications

\section{Figure Captions -}

1. A Pt adatom (labelled 1 ) in its ground state on the $\mathrm{Pt}(110)-1 \times 2$ missing row surface. According to the present calculations, its minimum energy diffusion path passes through one of the $h c p$ wall sites labelled $\mathbf{h}$, then through one of the adjacent $f c c$ sites labelled $\mathbf{f}$, and finally back to a channel hollow via another $h c p$ wall site.

2. Periodically repeated dimers in channels on the Pt(110) missing row surface. The dimers are the "doorway configuration" discussed in the text. Leapfrog diffusion occurs if adatom 1 moves to f', then between atoms $t_{2}$ and $\mathbf{2}$ to $f^{\prime \prime}$, to the doorway $h c p$ site, $\mathbf{h}$ " and finally to $\mathbf{c}$ ". Dissociation occurs if, as adatom 2 remains more or less fixed, adatom 1 moves to the $f c c$ hollow labeled $\mathbf{f}$. An alternate dissociation path corresponds to adatom 1 moving from its initial site, $\mathbf{c}$ directly along the channel to site $\mathrm{d}$. The dashed rectangle delineates the $4 \times 4$ supercell used in the present calculations. 
For Physical Review B, Rapid Comunications

\section{Table Captions -}

1. Dimer energy vs. the position of atom 1 as it dissociates from atom 2 . To keep the dissociation energy low, the latter remains close to its initial channel hollow site.

2. Computed energy vs. site for a Pt adatom on Pt(110)-1x2.

3. Dimer energy vs. the position of atom 1 along the leapfrog diffusion MEP. Keeping the total energy low, the latter remains close to its initial channel hollow site.

4. $\mathrm{CO}$ effect on the energy needed to dissociate a Pt dimer on Pt(110)-1x2 
Table 1:

\begin{tabular}{|c|c|}
\hline Atom 1 site & $E_{\text {diss }}(\mathrm{eV})$ \\
\hline channel hollow & 0.0 \\
\hline hollow to doorway barrier & 0.76 \\
\hline doorway configuration & 0.67 \\
\hline doorway to fcc barrier & 1.13 \\
\hline wall fcc & 0.90 \\
\hline & \\
\hline channel 2-fold & 1.24 \\
\hline 2nd-nbr channel hollow & 0.21 \\
\hline 4th-nbr channel hollow & 0.21 \\
\hline
\end{tabular}


For Physical Review B, Rapid Comunications

Table 2:

\begin{tabular}{|c|c|c|}
\hline Site & description & $\begin{array}{c}\text { Energy } \\
(\mathrm{eV})\end{array}$ \\
\hline channel hollow & depicted in Fig. 2 & 0.0 \\
\hline 5-fold to $h c p$ barrier & & 0.90 \\
\hline$h c p$ wall & label $\boldsymbol{h}$ in Fig. 2 & 0.84 \\
\hline$h c p$ to $f c c$ barrier & & 0.92 \\
\hline$f c c$ wall & label $\mathbf{f}$ in Fig. 2 & 0.67 \\
\hline 2-fold channel & depicted in Fig. 3b & 1.03 \\
\hline
\end{tabular}

Table 3:

\begin{tabular}{|c|c|c|}
\hline Atom 1 site & description & $\begin{array}{c}\text { Energy } \\
(\mathrm{eV})\end{array}$ \\
\hline channel hollow & directly above c in Fig. 1 & 0.0 \\
\hline hollow to "doorway" barrier & & 0.76 \\
\hline$h c p$ "doorway" & as depicted in Fig. 1 & 0.67 \\
\hline wall fcc & at label f' in Fig. 1 & 0.31 \\
\hline between wall \& atom 2 & as depicted in Fig. 3a & 0.72 \\
\hline
\end{tabular}


For Physical Review B, Rapid Comunications

Table 4:

\begin{tabular}{|c|c|c|}
\hline \# of CO's & CO site & $E_{\text {diss }}(\mathrm{meV})$ \\
\hline 0 & NA & 210 \\
\hline 1 & atop & 73 \\
\hline 1 & bridge & -41 \\
\hline 2 & atop, atop & -127 \\
\hline
\end{tabular}


For Physical Review B, Rapid Comunications

Fig. 1

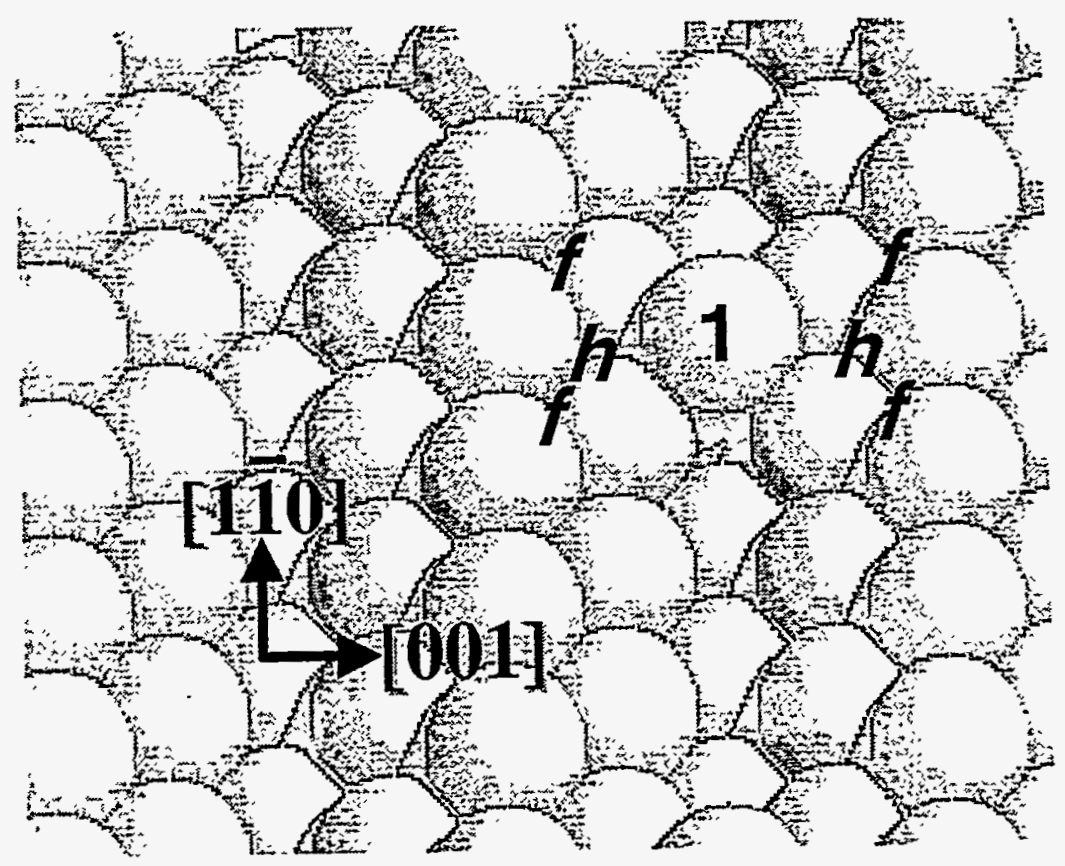


For Phy sical Review B, Rapid Comunications

Fig. 2

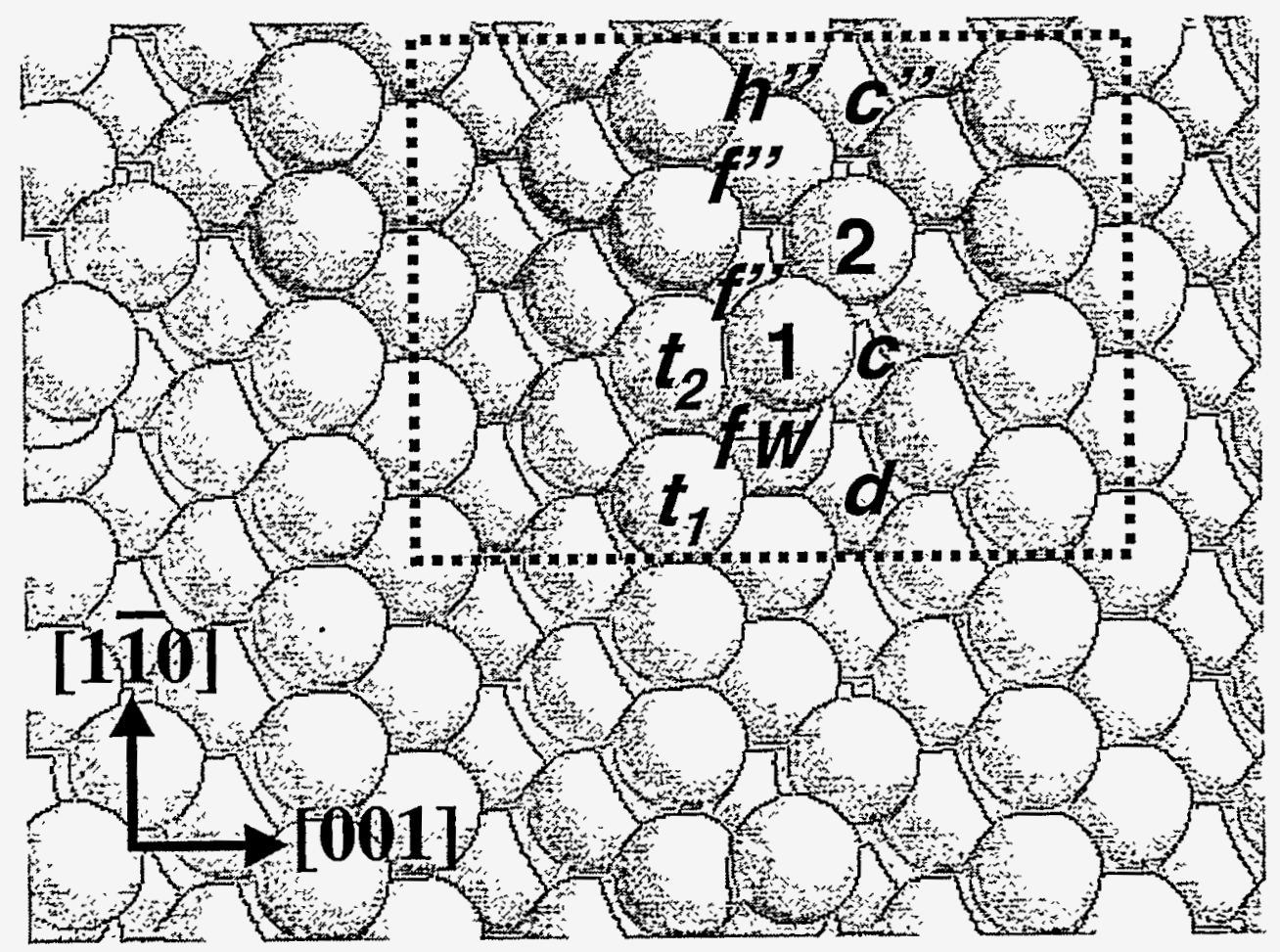

